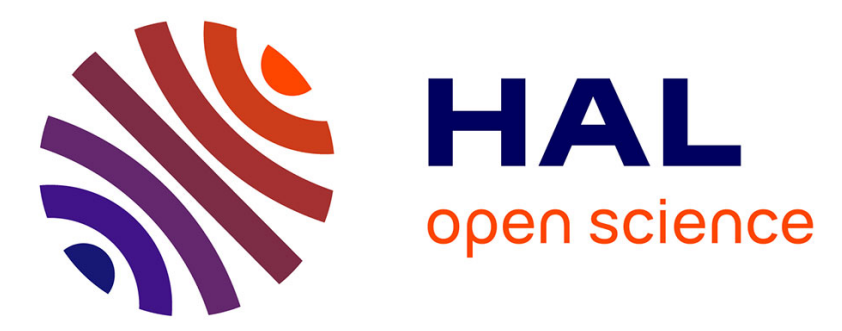

\title{
Patient-Specific Computational Analysis of Ventricular Mechanics in Pulmonary Arterial Hypertension
}

Ce Xi, Candace Latnie, Ju Le Tan, Samuel T. Wall, Martin Genet, Liang Zhong, Lik Chuan Lee, Xiaodan Zhao

\section{To cite this version:}

Ce Xi, Candace Latnie, Ju Le Tan, Samuel T. Wall, Martin Genet, et al.. Patient-Specific Computational Analysis of Ventricular Mechanics in Pulmonary Arterial Hypertension. Journal of Biomechanical Engineering, 2016, 138 (11), 10.1115/1.4034559 . hal-01427545

\section{HAL Id: hal-01427545 \\ https://hal.science/hal-01427545}

Submitted on 5 Jan 2017

HAL is a multi-disciplinary open access archive for the deposit and dissemination of scientific research documents, whether they are published or not. The documents may come from teaching and research institutions in France or abroad, or from public or private research centers.
L'archive ouverte pluridisciplinaire HAL, est destinée au dépôt et à la diffusion de documents scientifiques de niveau recherche, publiés ou non, émanant des établissements d'enseignement et de recherche français ou étrangers, des laboratoires publics ou privés. 


\title{
Patient-specific computational modeling of ventricular mechanics in pulmonary arterial hypertension
}

\author{
Xi $\mathrm{C}^{1}$, Latnie $\mathrm{C}^{1}$, Tan $\mathrm{JL}^{2}$, Wall $\mathrm{ST}^{3}$, Genet $\mathrm{M}^{4}$, Zhong $\mathrm{L}^{2,5}$, and Lee $\mathrm{LC}^{1, \dagger}$ \\ ${ }^{1}$ Department of Mechanical Engineering, Michigan State University, East Lansing, U.S.A. \\ ${ }^{2}$ National Heart Center Singapore, Singapore \\ ${ }^{3}$ Simula Research Laboratory, Oslo, Norway \\ ${ }^{4}$ Ecole Polytechnique, Palaiseau, France \\ ${ }^{5}$ Duke-NUS Medical School, Singapore \\ ${ }^{\dagger}$ Corresponding author: lclee@egr.msu.edu
}

May 10, 2016

\begin{abstract}
Patient-specific biventricular computational models associated with a normal subject and a patient diagnosed with pulmonary arterial hypertension (PAH) were developed to investigate the effects of this disease on ventricular mechanics. These models were developed using geometry reconstructed from magnetic resonance (MR) images, and constitutive descriptors of passive and active mechanics. Model parameter values associated with ventricular mechanical properties and myofiber architecture were obtained by fitting the models with the corresponding measured pressure-volume loops and the circumferential strain calculated from the MR images using a hyperelastic warping method. Our results shows that the peak right ventricle (RV) pressure was substantially higher in the PAH patient when compared to the normal (65 mmHg vs. $20 \mathrm{mmHg}$ ). Circumferential strain $\left(E_{c c}\right)$ and ejection fraction (EF) were comparatively lower in both the left ventricle (LV) and RV of the PAH patient (LV EF: $39 \%$ vs. $66 \%$ and RV EF: $18 \%$ vs. $64 \%$; LV $E_{c c}$ : $-2.1 \%$ vs $-9.4 \%$ and RV $E_{c c}-6.8 \%$ vs. $\left.-13.2 \%\right)$. On the other hand, passive stiffness, contractility and myofiber stress were all found to be substantially increased in the PAH patient in both the RV and the left ventricle (LV). Septum in the PAH patient was also found to possess a smaller curvature than the LV free wall. Simulations using the PAH model with varying RV preload and afterload revealed an approximately linear relationship between the septum curvature and the transseptal pressure gradient at early-diastole and end-systole, respectively. These findings suggest that PAH can induce LV remodeling and measurements of septum curvature may be useful in quantifying the transseptal pressure gradient in $\mathrm{PAH}$ patients.
\end{abstract}




\section{Introduction}

Pulmonary arterial hypertension (PAH) is a disease associated with vasoconstriction of the pulmonary arterial vessels that results in an elevated pulmonary arterial pressure. The clinical diagnosis for PAH is an elevated mean pulmonary artery (PA) pressure greater than $25 \mathrm{mmHg}$ with normal wedge pressure ${ }^{1}$. Without treatment, PAH can quickly lead to decompensated right heart failure and death. The current prognosis of PAH remains poor with about $15 \%$ mortality within 1 year on modern therapy ${ }^{2}$, and a low 3 -year survival rate of $67 \%^{3}$.

The elevated afterload in the right ventricle (RV) caused by PAH can lead to changes in ventricular mechanics ${ }^{4}$. For example, elevated RV pressure (RVP) can lead to abnormal ventricular deformation in the form of a "left ventricular septal bow" (LVSB), which is a leftward motion of the septum into the left ventricle (LV) cavity. Moreover, PAH is also associated with long-term functional, structural and geometrical changes in the RV ${ }^{5}$. These changes and their impacts on ventricular mechanics are currently not well-understood ${ }^{6}$. Although animal models have been used to understand ventricular mechanics associated with RV remodeling in $\mathrm{PAH}^{7,8}$, similar studies in humans are lacking and most clinical investigations have been confined to quantifying global ventricular mechanics through pressure-volume (PV) loop measurements ${ }^{9,10}$.

Image-based computational models are increasingly used to analyze ventricular mechanics in heart diseases (e.g., myocardial infarction ${ }^{11}$, left branch bundle block ${ }^{12}$, mitral valve regurgitation ${ }^{13,14}$ ) as well as heart failure treatments ${ }^{15}$. The focus of these computational analyses is, however, on the left ventricle (LV) that has historically received more attention than the RV. On the other hand, computational modeling analyses of PAH have mostly been performed on pulmonary arteries ${ }^{16,17}$ while the RV, whose function is a major determinant of prognosis in $\mathrm{PAH}^{18}$, has largely been overlooked.

To fill the void in the current research of PAH, we describe in here an image-based computational analysis of the ventricular mechanics in a PAH patient. Specifically, we seek to quantify regional ventricular myofiber stresses, myofiber strain, contractility and passive tissue stiffness in a PAH patient, and compare them to those found in an age- and gendermatched healthy human subject. We also use the PAH computational model to investigate and quantify the effects of interventricular pressure gradients on the septum curvature.

\section{Method}

\subsection{Acquisition}

A 3 T Philips scanner with ECG gating was used to acquire cine magnetic resonance (MR) images from one PAH patient as well as one gender- and age- matched normal human subject. Left and right ventricular, atria and arterial pressure were also acquired from the PAH patient by right heart catheterization as part of their clinical care. All data were acquired in the National Heart Center of Singapore and all enrolled participants gave written informed consent. 


\subsection{Biventricular geometry}

Three-dimensional biventricular geometries of the PAH patient and normal subject were reconstructed by manually delineating the contours of the LV endocardium, RV endocardium and epicardium in different short and long-axis views in the cine MR images. These contours were then fitted to obtain 3D surfaces of the LV endocardial, RV endocardial and epicardial surfaces using the medical images analysis software MeVisLab (http://www.mevislab.de). Finite element (FE) meshes for the two cases were generated in the volume enclosed by these surfaces using $\mathrm{GMSH}^{19}$. The meshes consist of approximately 4000 quadratic tetrahedral elements and 7000 nodes.

\subsection{Pressure volume loops}

Cavity volumes of the LV and RV were measured in the PAH patient and normal subject at different time frames within a cardiac cycle using the MR-reconstructed biventricular geometries described in the previous section. These measured volumes were used together with the acquired pressure data to reconstruct the LV and RV PV loops of the PAH patient. For the normal subject, normal right ventricular pressure waveform from a previous study 20 and a scaled normal left ventricular pressure waveform (with end-systolic pressure equal to 0.9 of the measured cuff pressure ${ }^{21}$ ) were used together with the cavity volume measurements to reconstruct the PV loops.

\subsection{Strain Analysis of MR images}

Regional circumferential strain was estimated from the PAH patient and the normal subject using hyperelastic warping in FEBio (http://www.febio.org) with the FEWarp plugin. Briefly, in hyperelastic warping, a FE model of the biventricular geometry from the template image was deformed into alignment with the corresponding object in the target image via a computed image-based local body force term that depends on (1) the difference in image intensity between the template and target images, (2) the target image intensity gradient and (3) a prescribed penalty factor ${ }^{22}$. In our analysis, the penalty factor was increased incrementally until the strain field converged. Spatially-averaged circumferential strain was computed in the LV and the RVFW at different cardiac time points using end-diastole as the reference configuration. Circumferential direction was prescribed using the Laplace-Dirichlet Rule-Based algorithm ${ }^{23}$ with myofiber angle set to be zero.

\subsection{Biventricular Finite Element Model}

Biventricular geometries from the corresponding MR images acquired at early diastole, in which the LV and RV pressures were at their lowest, were used as the reference geometries for the biventricular FE models of the PAH patient and normal subject (Figure 1a). Myocardial fiber orientation was prescribed in the models using a Laplace-Dirichlet Rule-Based algorithm ${ }^{23}$. Based previous experimental measurements ${ }^{24}$, myofiber helix angle was prescribed in the $\mathrm{LV}$ with a linear transmural variation from $60^{\circ}$ at the endocardium to $-60^{\circ}$ at 
the epicardium (i.e., $60^{\circ} /-60^{\circ}$ ) (Figure 1b). Given the lack of human RV myofiber data, myofiber orientation in the RVFW was described similarly using a linear transmural variation $\beta^{\circ} /-\beta^{\circ}$, in which $\beta$ is a model parameter found from fitting the clinical measurements.

\section{Cardiac Mechanics}

A cellular-based cardiac electromechanics model ${ }^{25,26}$ was used to model the mechanical behavior of the biventricular unit over a cardiac cycle. Because the focus here is on biventricular mechanics, we have simplified the model by assuming a homogeneous activation in the biventricular unit. The resultant model can therefore be described by the following system of ordinary differential equations (ODEs) and partial differential equations (PDEs):

$$
\begin{gathered}
\frac{\partial \mathbf{s}}{\partial t}=f(v, \mathbf{s}, \lambda) \\
\frac{\partial v}{\partial t}+I_{i o n}(v, \mathbf{s}, \lambda)=I_{s} \\
\nabla \cdot \boldsymbol{\sigma}=0
\end{gathered}
$$

Briefly, Eq. (1a) and (1b) consist of a system of ODEs that describe the local coupling between cellular electrophysiology ${ }^{27}$ and cross-bridge cycling process ${ }^{28}$. Here, s, $v, I_{\text {ion }}$ and $\lambda$ denote a vector of state variables consisting of various membrane channels and intracellular ionic concentration, the total ionic current that is scaled with the membrane capacitance, the transmembrane potential and the myofiber stretch, respectively. A stimulus current $I_{s}$ was prescribed over a short time interval to excite the biventricular unit.

Equation (1c) describes the mechanical equlibrium of the cardiac tissue with $\boldsymbol{\sigma}$ denoting the Cauchy stress tensor. An active stress formulation was used to model the contraction of the tissue. In this formulation, $\boldsymbol{\sigma}$ was decomposed into a passive component $\boldsymbol{\sigma}_{p}$ and an active component $\boldsymbol{\sigma}_{a}$ that accounts for the stiffening of the tissue during cross-bridge cycling, i.e.,

$$
\boldsymbol{\sigma}=\boldsymbol{\sigma}_{p}+\boldsymbol{\sigma}_{a}\left(\mathbf{s}, \lambda, \dot{\lambda} ; T_{r e f}\right) .
$$

The active stress tensor $\boldsymbol{\sigma}_{a}$ depends on the time evolution of the state variables $\mathbf{s}$, myofiber stretch $\lambda$, rate of elastic myofiber stretch $\dot{\lambda}$. The contractile stress was scaled by a reference tension $T_{r e f}$, which a model parameter that is associated with the tissue contractility. The passive stress $\boldsymbol{\sigma}_{p}$ was described by a Fung-type transversely isotropic hyperelastic constitutive model with the following strain energy function:

$$
\Psi=\frac{1}{2} C\left(e^{Q}-1\right)+C_{c o m p r}(J \ln J-J+1)
$$

where

$$
\begin{aligned}
Q & =b_{f f} E_{f f}^{2}+b_{x x}\left(E_{s s}^{2}+E_{n n}^{2}+E_{s n}^{2}+E_{n s}^{2}\right) \\
& +b_{f x}\left(E_{f n}^{2}+E_{n f}^{2}+E_{f s}^{2}+E_{s f}^{2}\right)
\end{aligned}
$$


In the above equation, $\mathbf{E}=\frac{1}{2}\left(\mathbf{F}^{\mathbf{T}} \mathbf{F}-\mathbf{I}\right)$ is the Green-Lagrange strain tensor, $J$ is the Jacobian of the deformation gradient tensor $\mathbf{F}$, and $E_{i j}$ with $i, j \in(f, s, n)$ are the strain components with $f, s, n$ respectively denoting the fiber, sheet and sheet normal directions. The passive material parameters are $C_{c o m p r}, C, b_{f f}, b_{x x} b_{f x}$. The passive stress $\boldsymbol{\sigma}_{p}$ is related to the strain energy function as follows:

$$
\boldsymbol{\sigma}_{p}=\frac{1}{J} \mathbf{F} \frac{\partial \mathbf{\Psi}}{\partial \mathbf{E}} \mathbf{F}^{\mathbf{T}}
$$

\section{Simulation of a cardiac cycle}

A full cardiac cycle was simulated based on an open-loop circulatory model. Specifically, passive filling was simulated by incrementally applying pressure to the LV and RV endocardial surfaces until the prescribed end-diastolic pressures (EDP) were reached. The biventricular model was then stimulated, and the LV and RV cavity volumes were constrained to remain constant during the isovolumic contraction phase. The ejection phase was simulated by coupling the LV and RV cavity volumes each to a 3-element Windkessel model when the LV and RV pressures exceeded the aortic and pulmonary valve opening pressures, respectively (Figure 1c). The isovolumic relaxation phase was simulated by constraining the cavity volumes and begins when outflows from the LV and RV became negative.

\section{Model parameterization}

Biventricular FE models of the PAH patient and normal subject were each divided into two material regions, namely, the LV and the RVFW with different passive stiffness $C$ and contractility $T_{\text {ref }}$ (Figure 1a). Together with the Windkessel model peripheral resistance and the RVFW myofiber helix angle $\beta$, these material parameters were manually adjusted to fit the (1) experimentally measured PV loops in the LV and RV, and (2) the regional circumferential strain measurements from hyperelastic warping in each case. All other material parameters were prescribed with the same values in both cases. Parameter values in the active contraction model were similar to those in Rice et al. ${ }^{28}$ whereas those in the passive strain energy function (i.e., $C_{c o m p r}, b_{f f}, b_{f x}$, and $b_{x x}$ ) were similar to those in Genet et al. ${ }^{29}$.

\section{Septum curvature}

The fitted PAH model was used to quantify the effects of varying RV afterload and preload on the regional curvature in the LV endocardial surface. Regional curvature $\kappa$ was calculated at the mid-ventricular level (half-way between the apex to base) from the biventricular FE model at different time point within a cardiac cycle. Specifically, contour of LV endocardial slice at the mid-ventricular level was parameterized as $r(\theta)$ in a polar coordinate system (Figure 1d) and $\kappa$ was computed at each point in the contour using:

$$
\kappa(\theta)=\frac{r^{2}+2 r^{\prime 2}-r r^{\prime \prime}}{\left(r^{2}+r^{\prime 2}\right)^{\frac{3}{2}}},
$$

where $r^{\prime} \equiv d r / d \theta$. Septum and LV free wall (LVFW) curvature were computed by averaging $\kappa$ for all the points that are located in their respective region. 


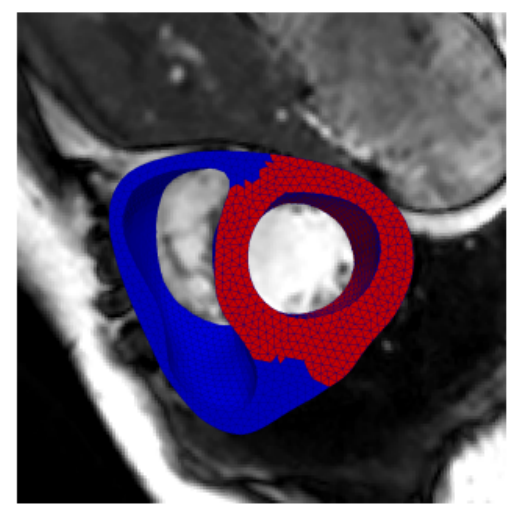

(a)

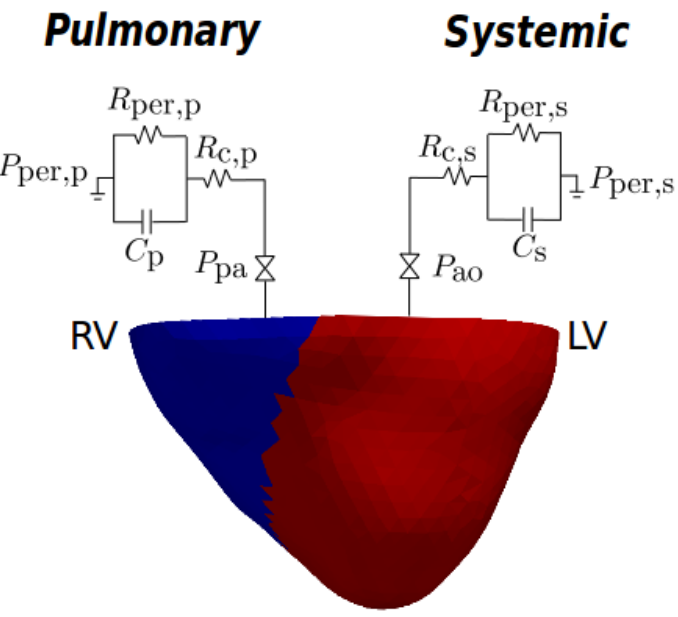

(c)

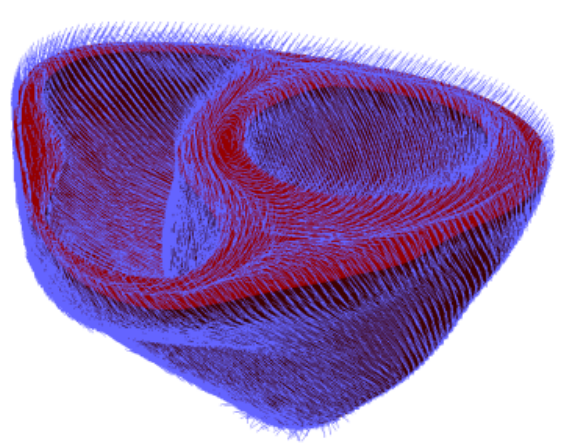

(b)

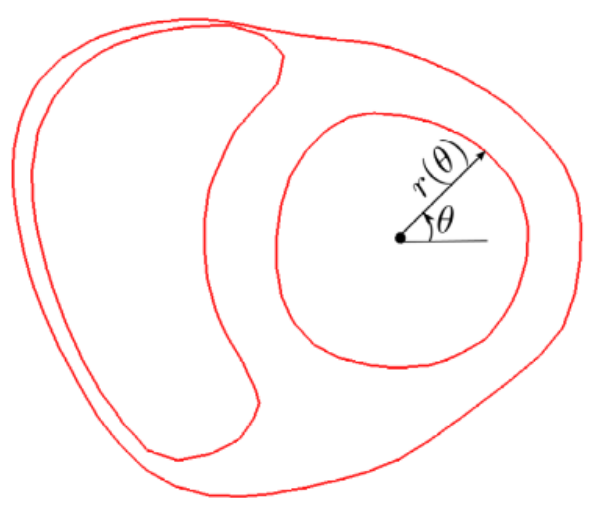

(d)

Figure 1: (a) 3D Biventricular geometry reconstructed from MR images with LV and RVFW material regions. (b) Myofiber orientation prescribed using LDRB method with a transmural variation of $60^{\circ}$ (endo) to $-60^{\circ}$ (epi) for the entire model. (c) Coupling the biventricular model to 3 -element Windkessel models. (d) Polar coordinate used to calculate local curvature $\kappa$ in the LV endocardium.

\section{Results}

\subsection{Pressure-volume loops}

The model-predicted PV loops of the RV and LV were in close agreements with the measurements in both PAH and normal cases (Figure 2). The differences between measurements and the model predictions were within 1\%. Peak RV pressure was about 3.5 times higher in the PAH patient than that found in the normal subject (65 $\mathrm{mmHg}$ vs. $20 \mathrm{mmHg}$ ), whereas peak LV pressure was only slightly higher in the PAH patient (110 $\mathrm{mmHg}$ vs. $100 \mathrm{mmHg}$ ). End-diastolic volume (EDV) and end-systolic volume (ESV) of the LV and RV were also larger in the PAH patient than in the normal subject with the largest difference found the RV (RV EDV: $275 \mathrm{ml}$ vs. $100 \mathrm{ml}$; RV ESV: $225 \mathrm{ml}$ vs. 36ml). Correspondingly, the ejection 
fraction (EF) of the PAH patient was substantially lower when compared to that of the normal subject (LV EF: $39 \%$ vs. $66 \%$ and RV EF: $18 \%$ vs. $64 \%$ ).

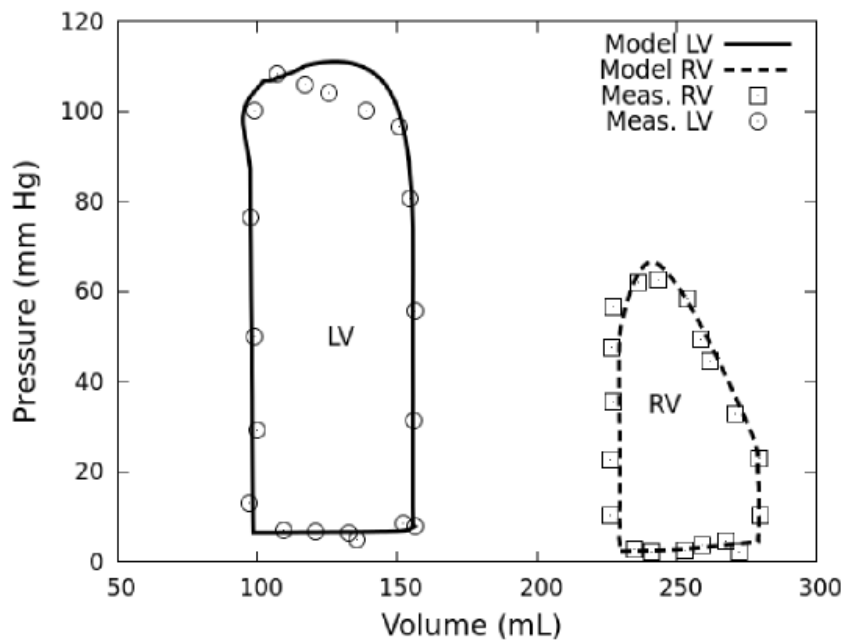

(a)

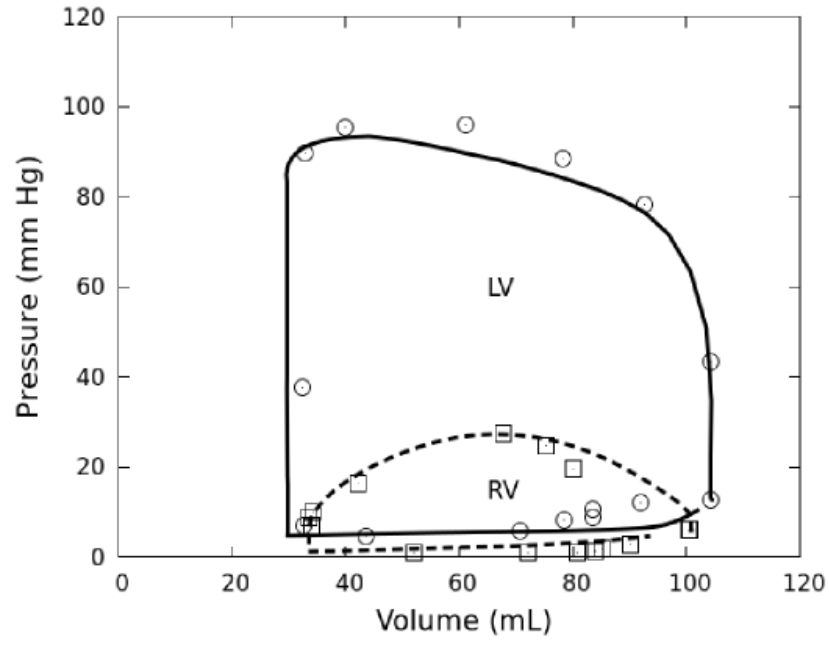

(b)

Figure 2: Measurements and model prediction of the PV loops for the (a) PAH case and (b) normal case.

\subsection{Regional circumferential strain}

Circumferential strain measured using hyperelastic warping was substantially depressed in both LV and RVFW of the PAH case when compared to the normal case (Figure 3a, b). Specifically, the spatially-averaged peak circumferential strain in a cardiac cycle was $-2.1 \%$ and $-9.4 \%$ in the RVFW of the PAH and the normal cases, respectively. Left ventricular peak circumferential strain was also depressed in the PAH patient when compared to the normal subject (-6.8\% vs. $-13.2 \%)$. In both cases, peak circumferential strain in the LV was found to be larger than that in the RVFW.

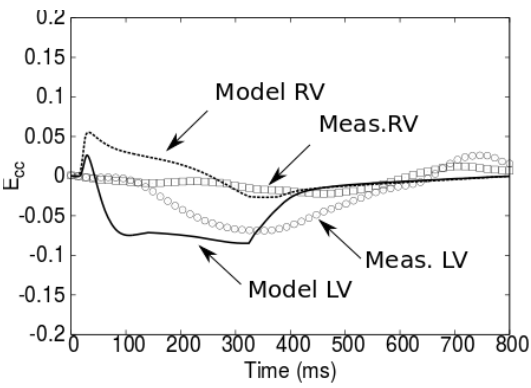

(a)

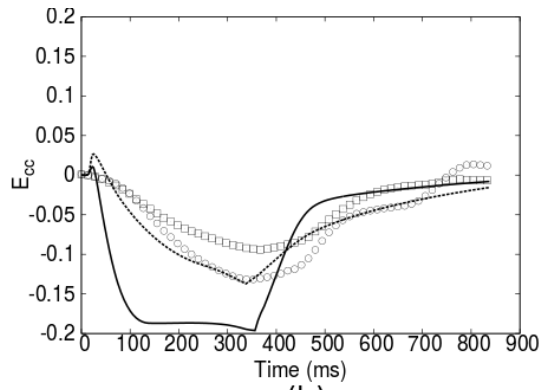

(b)

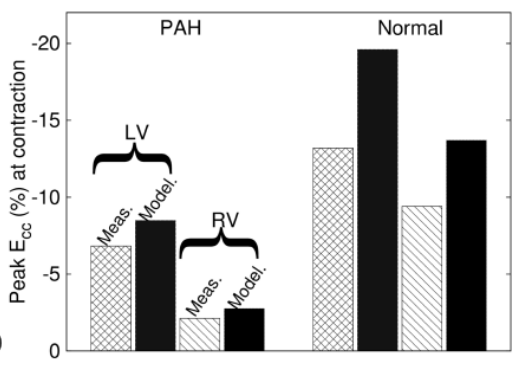

(c)

Figure 3: Circumferential strain $E_{c c}$ for the (a): PAH and (b): normal case. (c): Comparision of regional peak negative $E_{c c}$ between $\mathrm{PAH}$ and normal cases.

The model predictions agreed reasonably well with these strain measurements, with maximum differences between the model-predicted peak strain and the measurements at about 
$5 \%$ in the LV of the normal subject. We also found that the circumferential strain is very sensitive to the fiber orientation, and in order to capture the differences in strain between the LV and RVFW, transmural variation of myofiber angle in the RVFW has to be larger than that in the LV.

\subsection{Myofiber stress}

Myofiber stress in the PAH patient was substantially higher than that of the normal subject in the entire biventricular region, with regions of high myofiber stress found in the LVFW and septum (Figure 4). Specifically, peak average myofiber stress in the RVFW and LV of the PAH patient were almost twice the values found in the normal subject (LV: 99.7kPa vs. $51.2 \mathrm{kPa}$; RVFW: $73.2 \mathrm{kPa}$ vs. $43 \mathrm{kPa}$ ). For both of the PAH patient and the normal subject, the stress in the LV is higher than that in the RVFW.
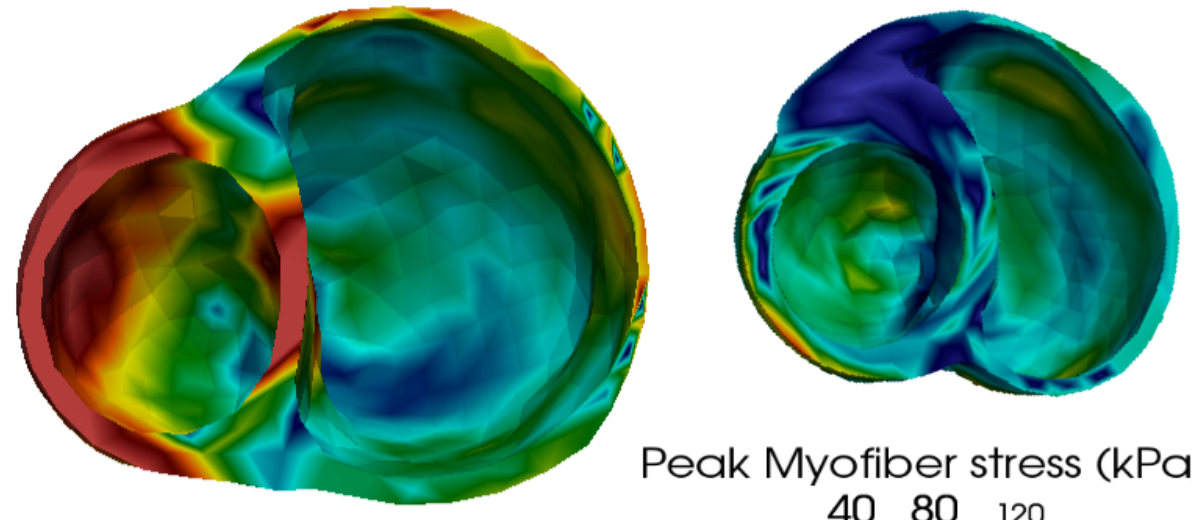

Peak Myofiber stress (kPa)

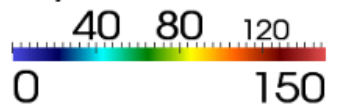

Figure 4: Peak myofiber stress in the PAH patient (left) and the normal subject (right).

\subsection{Model parameters}

Table I: Regional Model Parameters: Passive Stiffness $C$ (kpa), Myocardial Contractility $T_{r e f}(\mathrm{kpa})$, Peripheral Resistance $R_{\text {per }}(\mathrm{mmHg} \cdot \mathrm{min} / \mathrm{mL})$

\begin{tabular}{cccccccc}
\hline \hline \multirow{2}{*}{ Case } & \multicolumn{3}{c}{ LV } & & \multicolumn{4}{c}{ RV } \\
& $C$ & $T_{\text {ref }}$ & $R_{\text {per, s }}$ & $C$ & $T_{\text {ref }}$ & $\beta$ & $R_{\text {per, }}$ \\
\hline PAH & 1.68 & 262.5 & 0.005 & 1.96 & 160 & $80^{\circ}$ & 0.00325 \\
Normal & 0.035 & 123 & 0.005 & 0.12 & 60 & $75^{\circ}$ & 0.000625 \\
\hline
\end{tabular}

Table I shows the model parameters for the PAH patient and the normal subject that were calibrated to against the PV loops and the regional circumferential strains. The model parameters $C$ and $T_{\text {ref }}$ reflects the tissue's passive stiffness and contractility, respectively. 
We found that the tissue's passive stiffness and contractility were both higher in the LV and RV of the PAH patient than those in the normal subject. Peripheral resistance in the RV was also substantially higher in the PAH patient than in the normal subject, but was similar in the LV of both. We also found that the transmural variation of myofiber angle was steeper in the RV with the fibers oriented more longitudinally than in the LV in both cases. This regional variation was found necessary to produce a smaller circumferential strain in the RV (compared to the LV) as found in the hyperelastic warping measurements (Figure 3).

\subsection{Septum curvature}

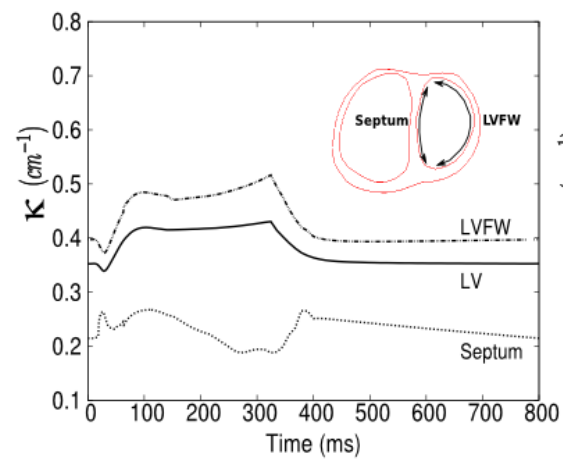

(a)

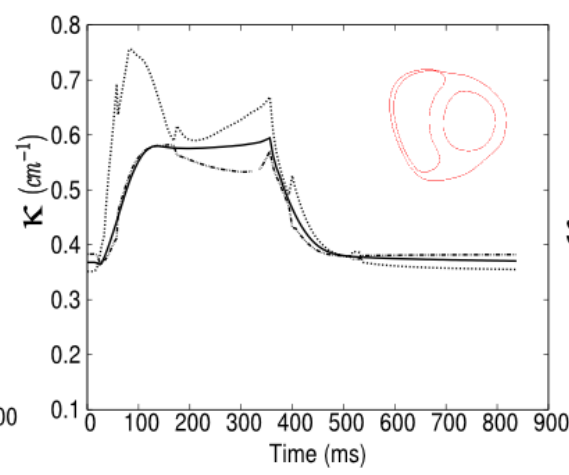

(b)

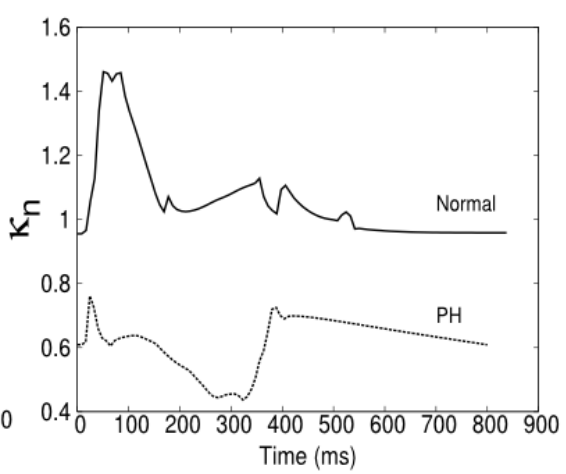

(c)

Figure 5: Curvature $\kappa$ in the LV endocardial surface of (a) PAH patient and (b) normal subject. (c) Comparison of normalized curvature $\kappa_{n}$ between PAH patient and normal subject.

Two-dimensional curvature $\kappa$ calculated from Eq. (5) on the LV endocardial surface at the mid-ventricular short-axis slice shows a substantially lower $\kappa$ in the septum of the PAH patient (Figure 5). Compared to the normal subject model, which has a homogeneous curvature in the LV, the septum curvature in the PAH patient was substantially lower than that found in the LVFW. To eliminate the effects of LV diameter on $\kappa$, the septum curvature was normalized against the average LV curvature to give a normalized curvature $\kappa_{n}$. We found that the normalized curvature $\kappa_{n}$ was lower in the septum of the PAH patient than that in the normal subject.

\section{Effects of afterload}

The septum curvature $\kappa$ decreased with increasing RV afterload (Figure 6a), which was adjusted in the PAH model by simultaneously increasing the pulmonary peripheral resistance $R_{\text {per,p }}$ and decreasing the RVFW contractiliy $T_{\text {ref }}$ so that the stroke volume was relatively unchanged (Figure 6c). The largest variation of septum $\kappa$ with RV afterload occurs primarily during the systole phase, with the maximum curvature $\kappa$ varying almost linearly $(r=0.984)$ with the end-systolic transseptal pressure gradient between the LV and RV (i.e., LVP - RVP) (Figure 6b). On the other hand, we found that the curvature $\kappa$ was less than the normal case (Figure 5b) even after reducing the afterload in the PAH model to a value close to the normal physiological RV peak pressure $(30 \mathrm{mmHg})$. 


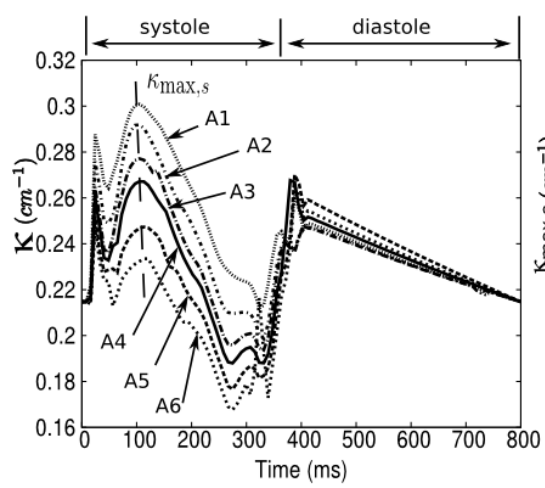

(a)

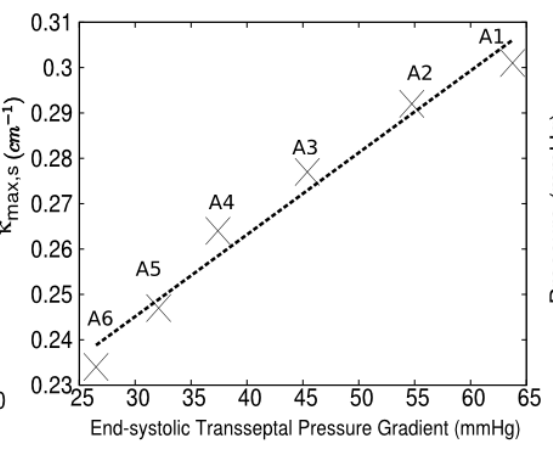

(b)

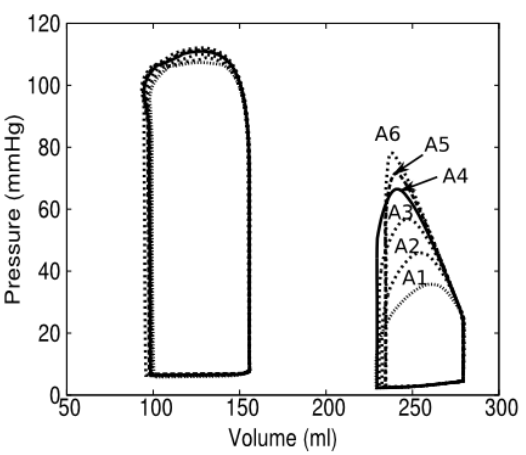

(c)

Figure 6: Effects of afterload on septum curvature within a cardiac cycle. (a): Time-course of $\kappa$ with different RV afterload. (b): End-systolic curvature $\kappa \max , s$ vs. end-systolic transseptal pressure gradient. (c): Corresponding PV loops.

\section{Effects of preload}

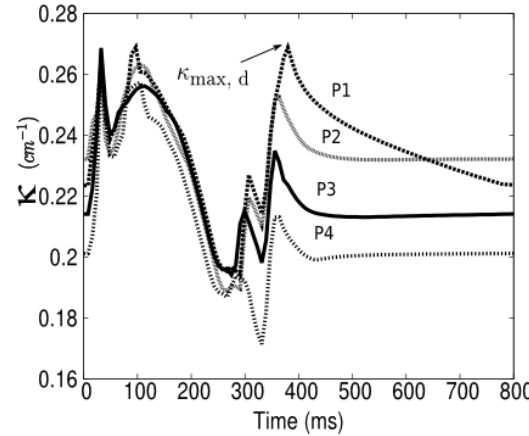

(a)

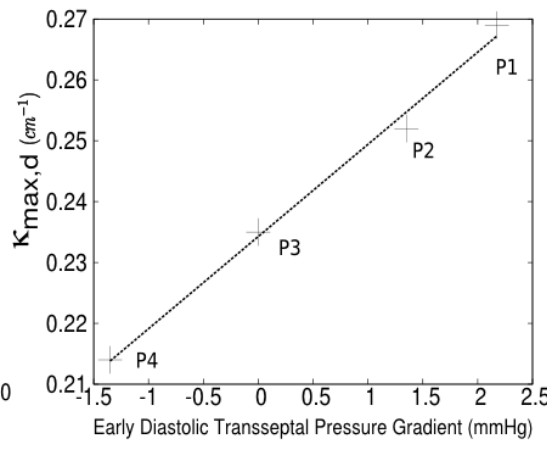

(b)

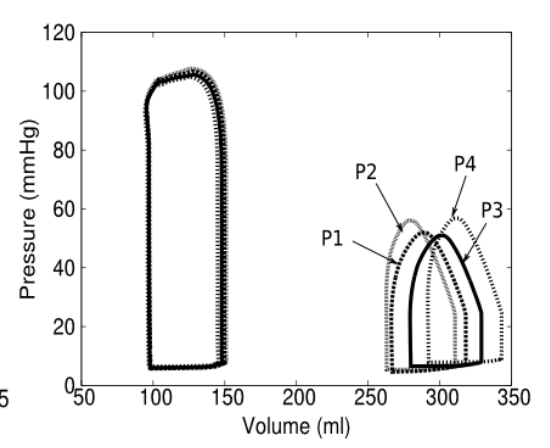

(c)

Figure 7: Effects of preload on septum curvature within a cardiac cycle. (a): Time-course of $\kappa$ with different transseptal pressure gradient. (b): Early-diastolic curvature $\kappa_{\max , d}$ vs. earlydiastolic transseptal pressure gradient. (c): Corresponding PV loops.

The septum curvature also decreased with increasing RV preload (Figure 7a), which was simulated by increasing the prescribed end-diastolic pressure. Due to length-dependent effects of the contraction model, RVFW contractiliy $T_{\text {ref }}$ was simultaneously adjusted to maintain a constant stroke volume in each of the different preload cases with respect to the baseline PAH case (Figure 7c). In contrast to the effects of afterload, the largest variation of septum curvature $\kappa$ occurs primarily during early diastole, whereas the curvature was little changed in systole. Interestingly, the maximum diastolic curvature $\kappa_{\max }$ seems to vary almost linearly $(r=0.997)$ with the transseptal pressure gradient of between LV and RV at early diastole. Specifically, we found that an increase in transseptal gradient by $1 \mathrm{~mm} \mathrm{Hg}$ produce an average decrease in the curvature by $0.0156 \mathrm{~cm}^{-1}$. 


\section{Discussion}

We have described the development of patient-specific biventricular computational models using pressure measurements and MR data from a PAH patient and a normal human subject. In each case, we have reconstructed the LV and RV PV loops using the pressure data and cavity volumes quantified from the MR images. A hyperelastic warping method was also used to compute the LV and RVFW circumferential strains from cine MR images. These strain measurements and PV loops were used to fit the biventricular computational models.

Using these fitted models, we have compared differences in the in vivo regional ventricular mechanics between the PAH patient and normal subject, which includes regional myofiber stress distribution, mechanical properties and regional curvature. Additionally, we have also quantify the effects of RV preload and afterload on the septal curvature using the PAH model. As most previous computational studies of PAH have focused on the arteries ${ }^{16,17}$, this study, to the best of our knowledge, is the first patient-specific computational analysis of the ventricular mechanics associated with PAH.

\subsection{Circumferential Strain}

Compared to the LV, quantitificaion of RV strain is significantly more challenging because of its complex geometry and thin wall. Nevertheless, RV strain analysis on PAH patients have been performed using speckle tracking on echocardiographic images ${ }^{30}$ as well as strainencoded MR imaging ${ }^{31}$. In these analyses, RV circumferential strain of PAH patients was found to be significantly depressed compared to normal subjects. Our results, which were obtained using a hyperelastic warping method ${ }^{22}$ on cine MR images, agree with these studies.

Besides a reduction in RV circumferential strain, we also found that the LV circumferential strain was reduced in the PAH patient compared to the normal subject $(-6.8 \%$ in PAH vs. $-13.2 \%$ in Normal). This feature was also found in another echocardiography study $^{32}$ in which both the LV lateral wall and septum circumferential strains were found to be significantly reduced by about $5 \%$ in pulmonary hypertensive patients.

\subsection{Myofiber Stress}

The use of Laplace's law to estimate RV wall stress is complicated by its irregular geometry ${ }^{33}$. Here, we have overcome this complication in our computational analysis, and have found a higher myofiber stress in the RVFW of the PAH patient that is about twice as high as that in the normal subject. The larger RVFW myofiber stress can be attributed to the combination of a higher afterload ( $3 \times$ of the normal) and a substantially bigger RV $(2 \times$ of the normal) in the PAH patient. In addition, we found that the LV myofiber stress was also about twice as high as that in the normal subject despite having a normal LV afterload. The higher LV myofiber stress could be attributed to the larger LV cavity $(1.5 \times$ of the normal). Given that elevated myofiber stress is feature that is associated with ventricular remodeling, this result suggests that the remodeling associated with PAH may not only be confined to the RV, but could also be found in the LV. 


\subsection{Fitted model parameters}

\section{Ventricular Properties}

The fitted model parameters associated with the regional biventricular mechanical properties also suggest significant remodeling in the PAH patient. Specifically, we found that the diastolic passive stiffness $C$ and the contractility $T_{r e f}$ of the PAH patient were larger than that of the normal subject in both LV and RVFW. These findings were consistent with previous experimental studies. By comparing the passive tension found in the RV tissue of PAH patients undergoing heart/lung transplantation and donors with non-failing heart, Rain et al. ${ }^{34}$ found that RV diastolic stiffness is increased in PAH patients and is closely associated with the disease severity measured using stroke volume and 6-minute walk test. They attributed this increase to RV fibrosis and the intrinsic stiffening of the RV myocytes. A similar finding was also reported in rats subjected to pulmonary artery banding ${ }^{7}$. Interestingly, the study by Rain et al. ${ }^{34}$ also found an increase in the RV end-systolic elastance and the force-generating capacity in isolated RV cardiomyocytes from PAH patients. An increase in RV end-systolic elastance was also found in rats induced with $\mathrm{PH}$ via monocrotaline injection compared to the controls ${ }^{35}$. As suggested in these studies, the increase in RV contractility associated with PAH is most likely a compensatory mechanism in the RV attempting to cope with the increased afterload.

That we found that the LV diastolic passive stiffness and contractility was also increased suggests the presence of remodeling in the LV of the PAH patient (as with our findings for circumferential strain and myofiber stress). Although we did not find any studies that measure LV mechanical properties directly associated PAH, it was observed that patients with right heart failure often have relaxation abnormalities that may affect the mechanical properties of the $\mathrm{LV}^{36}$. Moreover, recent studies have also found evidence of electrophysiological remodeling in the LV of PAH patients ${ }^{37}$.

\section{Myofiber orientation}

Histological studies have found that compared to the LV that has muscle fiber running obliquely towards the apex, the deep muscle fibers of the RV are longitudinally aligned from base to apex ${ }^{38}$. Our result also suggests that the myofiber orientation is more longitudinal in the RV than in the LV in both cases, an outcome that is in part due to the smaller circumferential strain found in the RVFW in both cases. On the other hand, studies on rats $^{7}$ have found that both myofiber and collagen orientations in the RVFW became more longitudinal as a result of RV remodeling caused by PAH. We, however, did not find this feature in our study.

\subsection{Septum Curvature}

The fact that abnormal septal geometry and motion is a well-recognized feature in PAH patients ${ }^{39}$ has motivated us to quantify this feature, as well as investigate its sensitivity to RV preload and afterload. Our results show that the septum curvature from the short axis

view at mid-ventricular level was substantially lower in the PAH patient compared to that in the normal subject, which implies a more "flattened" septum. 
With varying RV preload (and constant RV afterload), we found that the septum curvature at early-diastole increased linearly with increasing diastolic transseptal pressure gradient (LVP-RVP) $(r=0.997)$. Septum curvature in systole is not much affected by changes in the preload. Similar findings were also reported in other studies ${ }^{40,41}$, which found that the septum shape and motion at early-diastole are sensitive to the transseptal pressure gradient between the LV and RV. Tanaka et al. ${ }^{40}$ found that diastolic "bowing" of septum toward the LV (negative curvature) in PAH patients resulted from a negative transseptal pressure gradient whereas Beyar et $\mathrm{al}^{41}$ reported that "bowing" occurs only at a transseptal pressure gradient lower than $-5 \mathrm{mmHg}$. Although we did not observe any "bowing" for the range of transseptal pressure gradient between -1.5 to $2.5 \mathrm{mmHg}$, our finding of a linear variation between the pressure gradient and septum curvature is consistent with these studies.

Similarly, we found that the end-systolic septum curvature increased almost linearly with increasing systolic transseptal pressure gradient $(r=0.984)$ while the curvature at diastole is insensitive to the variation in afterload. Our result is quantitatively in good agreement with an echocardiographic study on children with pulmonary hypertension ${ }^{42}$, which found a correlation of $r=0.86$ between septum curvature and RV end-systolic pressure. This direct relationship between septum curvature and end-systolic pressure would suggest that the curvature can potentially be used as a non-invasive marker for quantifying RV pressure in PAH patient.

\subsection{Critique of the model}

Overall, predictions of the biventricular computational models are generally consistent with the results from clinical and experimental studies of PAH. Our results have also suggest that PAH may produce significant LV remodeling though caution must be exercised in extrapolating these results, which were based only from one model. Additionally, there are also some limitations that are associated with our models.

First, we have used the biventricular geometry that corresponds to the lowest pressure at the filling phase as the zero-stress state, which may affect the values of the fitted mechanical properties. This, of course, is an approximation as the zero-stress state does not exist in vivo. Other investigators have used the end-systole ${ }^{43}$ or mid-systole ${ }^{44}$ as the zero-stress state, or have estimated the zero-stress state from the end-diastole geometry using prescribed mechanical properties ${ }^{12}$. To the best of our knowledge, there appears to be no consensus on the best approach to address this issue.

Second, we have not sampled the entire parameter space in fitting our model. Specifically, we have kept the passive tissue anisotropy fixed based on values obtained in normal humans ${ }^{29}$ and the LV myofiber orientation fixed at $60^{\circ} /-60^{\circ}$. We have done so in order to focus on the key features found in the RV that are associated with PAH.

\section{References}

[1] McLaughlin, V. V., Archer, S. L., Badesch, D. B., Barst, R. J., Farber, H. W., Lindner, J. R., Mathier, M. a., McGoon, M. D., Park, M. H., Rosenson, R. S., Rubin, L. J., Tapson, V. F., and Varga, J., 2009. "ACCF/AHA 2009 Expert Consensus Document on 
Pulmonary Hypertension. A Report of the American College of Cardiology Foundation Task Force on Expert Consensus Documents and the American Heart Association Developed in Collaboration With the American College o". J. Am. Coll. Cardiol., 53(17), pp. 1573-1619.

[2] Thenappan, T., Shah, S. J., Rich, S., and Gomberg-Maitland, M., 2007. "A USA-based registry for pulmonary arterial hypertension: 1982-2006". Eur. Respir. J., 30(6), pp. 1103-1110.

[3] Humbert, M., Sitbon, O., Ya??ci, A., Montani, D., O’Callaghan, D. S., Ja??s, X., Parent, F., Savale, L., Natali, D., G??nther, S., Chaouat, A., Chabot, F., Cordier, J. F., Habib, G., Gressin, V., Jing, Z. C., Souza, R., Simonneau, G., Yaïci, a., Montani, D., O'Callaghan, D. S., Jaïs, X., Parent, F., Savale, L., Natali, D., Günther, S., Chaouat, A., Chabot, F., Cordier, J. F., Habib, G., Gressin, V., Jing, Z. C., Souza, R., Simonneau, G., Ya??ci, A., Montani, D., O'Callaghan, D. S., Ja??s, X., Parent, F., Savale, L., Natali, D., G??nther, S., Chaouat, A., Chabot, F., Cordier, J. F., Habib, G., Gressin, V., Jing, Z. C., Souza, R., and Simonneau, G., 2010. "Survival in incident and prevalent cohorts of patients with pulmonary arterial hypertension". Eur. Respir. J., 36(3), pp. 549-555.

[4] Vonk Noordegraaf, a., Galiè, N., Galie, N., and Galiè, N., 2011. "The role of the right ventricle in pulmonary arterial hypertension." . Eur. Respir. Rev., 20(122), pp. 243-53.

[5] Jessup, M., Sutton, M. S., Weber, K. T., and Janicki, J. S., 1987. "The effect of chronic pulmonary hypertension on left ventricular size, function, and interventricular septal motion.". Am. Heart J., 113(5), pp. 1114-22.

[6] Borgdorff, M. a. J., Dickinson, M. G., Berger, R. M. F., and Bartelds, B., 2015. "Right ventricular failure due to chronic pressure load: What have we learned in animal models since the NIH working group statement?". Heart Fail. Rev., 20(4), pp. 475-491.

[7] Hill, M. R., Simon, M. A., Valdez-Jasso, D., Zhang, W., Champion, H. C., and Sacks, M. S., 2014. "Structural and Mechanical Adaptations of Right Ventricle Free Wall Myocardium to Pressure Overload". Ann. Biomed. Eng., 42(12), pp. 2451-2465.

[8] Wang, Z., Schreier, D. a., Hacker, T. a., and Chesler, N. C., 2013. "Progressive right ventricular functional and structural changes in a mouse model of pulmonary arterial hypertension". Physiol. Rep., 1, pp. n/a-n/a.

[9] McCabe, C., White, P. a., Hoole, S. P., Axell, R. G., Priest, A. N., Gopalan, D., Taboada, D., MacKenzie Ross, R., Morrell, N. W., Shapiro, L. M., and Pepke-Zaba, J., 2014. "Right ventricular dysfunction in chronic thromboembolic obstruction of the pulmonary artery: a pressure-volume study using the conductance catheter.". J. Appl. Physiol., 116(29), pp. 355-63.

[10] Trip, P., Rain, S., Handoko, M. L., van der Bruggen, C., Bogaard, H. J., Marcus, J. T., Boonstra, A., Westerhof, N., Vonk-Noordegraaf, A., and de Man, F. S., 2015. "Clinical relevance of right ventricular diastolic stiffness in pulmonary hypertension". Eur. Respir. J., pp. 1-10. 
[11] Lee, L. C., Wenk, J. F., Klepach, D., Zhang, Z., Saloner, D., Wallace, A. W., Ge, L., Ratcliffe, M. B., and Guccione, J. M., 2011. "A Novel Method for Quantifying In-Vivo Regional Left Ventricular Myocardial Contractility in the Border Zone of a Myocardial Infarction". J. Biomech. Eng., $133(9)$, p. 094506.

[12] Aguado-Sierra, J., Krishnamurthy, A., Villongco, C., Chuang, J., Howard, E., Gonzales, M. J., Omens, J., Krummen, D. E., Narayan, S., Kerckhoffs, R. C. P., and McCulloch, A. D., 2011. "Patient-specific modeling of dyssynchronous heart failure: A case study". Prog. Biophys. Mol. Biol., 107, pp. 147-155.

[13] Baillargeon, B., Costa, I., Leach, J. R., Lee, L. C., Genet, M., Toutain, A., Wenk, J. F., Rausch, M. K., Rebelo, N., Acevedo-Bolton, G., Kuhl, E., Navia, J. L., and Guccione, J. M., 2015. "Human Cardiac Function Simulator for the Optimal Design of a Novel Annuloplasty Ring with a Sub-valvular Element for Correction of Ischemic Mitral Regurgitation". Cardiovasc. Eng. Technol., 6(2), pp. 105-116.

[14] Wenk, J. F., Zhang, Z., Cheng, G., Malhotra, D., Acevedo-Bolton, G., Burger, M., Suzuki, T., Saloner, D. a., Wallace, A. W., Guccione, J. M., and Ratcliffe, M. B., 2010. "First finite element model of the left ventricle with mitral valve: insights into ischemic mitral regurgitation.". Ann. Thorac. Surg., 89(5), pp. 1546-1553.

[15] Lee, L. C., Wall, S. T., Genet, M., Hinson, A., and Guccione, J. M., 2014. "Bioinjection treatment: Effects of post-injection residual stress on left ventricular wall stress". J. Biomech., 47(12), pp. 3115-3119.

[16] Hunter, K. S., Feinstein, J. A., Ivy, D. D., and Shandas, R., 2010. "Computational simulation of the pulmonary arteries and its role in the study of pediatric pulmonary hypertension". Prog. Pediatr. Cardiol., 30(1-2), pp. 63-69.

[17] Kheyfets, V. O., Rios, L., Smith, T., Schroeder, T., Mueller, J., Murali, S., Lasorda, D., Zikos, A., Spotti, J., Reilly, J. J., and Finol, E. A., 2015. "Patient-specific computational modeling of blood flow in the pulmonary arterial circulation". Comput. Methods Programs Biomed., 120(2), pp. 88-101.

[18] Voelkel, N. F., Quaife, R. A., Leinwand, L. a., Barst, R. J., McGoon, M. D., Meldrum, D. R., Dupuis, J., Long, C. S., Rubin, L. J., Smart, F. W., Suzuki, Y. J., Gladwin, M., Denholm, E. M., and Gail, D. B., 2006. "Right ventricular function and failure: Report of a National Heart, Lung, and Blood Institute working group on cellular and molecular mechanisms of right heart failure". Circulation, 114(17), pp. 1883-1891.

[19] Geuzaine, C., and Remacle, J.-F. F., 2009. "Gmsh: A 3-D finite element mesh generator with built-in pre- and post-processing facilities". Int. J. Numer. Methods Eng., $79(11)$, Sept., pp. 1309-1331.

[20] Redington, A. N., Gray, H. H., Hodson, M. E., Rigby, M. L., and Oldershaw, P. J., 1988. "Characterisation of the normal right ventricular pressure-volume relation by biplane angiography and simultaneous micromanometer pressure measurements". Br Hear. J, 59(1), pp. 23-30. 
[21] Kelly, R. P., Ting, C. T., Yang, T. M., Liu, C. P., Maughan, W. L., Chang, M. S., and Kass, D. a., 1992. "Effective arterial elastance as index of arterial vascular load in humans.". Circulation, $8 \boldsymbol{8}(2)$, pp. 513-521.

[22] Veress, A. I., Gullberg, G. T., and Weiss, J. A., 2005. "Measurement of strain in the left ventricle during diastole with cine-MRI and deformable image registration.". J. Biomech. Eng., 127(7), pp. 1195-1207.

[23] Bayer, J. D., Blake, R. C., Plank, G., and Trayanova, N. A., 2012. "A novel rule-based algorithm for assigning myocardial fiber orientation to computational heart models". Ann. Biomed. Eng., 40(10), pp. 2243-2254.

[24] Streeter, D. D., Spotnitz, H. M., Patel, D. P., Ross, J., and Sonnenblick, E. H., 1969. "Fiber orientation in the canine left ventricle during diastole and systole.". Circ. Res., 24(3), Mar., pp. 339-347.

[25] Sundnes, J., Wall, S., Osnes, H., Thorvaldsen, T., and McCulloch, a. D., 2012. "Improved discretisation and linearisation of active tension in strongly coupled cardiac electro-mechanics simulations.". Comput. Methods Biomech. Biomed. Engin.(March 2013), July, pp. 37-41.

[26] Lee, L., Sundnes, J., Genet, M., Wenk, J., and S.T., W., 2015. "An Integrated Electromechanical-Reversible Growth Heart Model for Simulating Cardiac Therapies". Biomech. Model. Mechanobiol., p. (In press).

[27] Winslow, R. L., Rice, J., Jafri, S., Marban, E., and O'Rourke, B., 1999. "Mechanisms of Altered Excitation-Contraction Coupling in Canine Tachycardia-Induced Heart Failure, II : Model Studies". Circ. Res., 84(5), Mar., pp. 571-586.

[28] Rice, J. J., Wang, F., Bers, D. M., and de Tombe, P. P., 2008. "Approximate model of cooperative activation and crossbridge cycling in cardiac muscle using ordinary differential equations.". Biophys. J., 95(5), Sept., pp. 2368-90.

[29] Genet, M., Lee, L. C., Nguyen, R., Haraldsson, H., Acevedo-Bolton, G., Zhang, Z., Ge, L., Ordovas, K., Kozerke, S., and Guccione, J. M., 2014. "Distribution of normal human left ventricular myofiber stress at end-diastole and end-systole-a target for in silico studies of cardiac procedures". J. Appl. Physiol., 117, pp. 142-52.

[30] Smith, B. C. F., Dobson, G., Dawson, D., Charalampopoulos, A., Grapsa, J., and Nihoyannopoulos, P., 2014. "Three-dimensional speckle tracking of the right ventricle: Toward optimal quantification of right ventricular dysfunction in pulmonary hypertension". J. Am. Coll. Cardiol., 64(1), pp. 41-51.

[31] Oyama-Manabe, N., Sato, T., Tsujino, I., Kudo, K., Manabe, O., Kato, F., Osman, N. F., and Terae, S., 2013. "The strain-encoded (SENC) MR imaging for detection of global right ventricular dysfunction in pulmonary hypertension". Int. J. Cardiovasc. Imaging, 29(2), pp. 371-378. 
[32] Puwanant, S., Park, M., Popovi??, Z. B., Tang, W. H. W., Farha, S., George, D., Sharp, J., Puntawangkoon, J., Loyd, J. E., Erzurum, S. C., and Thomas, J. D., 2010. "Ventricular geometry, strain, and rotational mechanics in pulmonary hypertension". Circulation, 121(2), pp. 259-266.

[33] Bellofiore, A., and Chesler, N. C., 2013. Methods for measuring right ventricular function and hemodynamic coupling with the pulmonary vasculature.

[34] Rain, S., Handoko, L., Trip, P., Gan, T.-J., Westerhof, N., Stienen, G., Paulus, W., Ottenheijm, C., Marcus, T., Dorfmüller, P., Guignabert, C., Humbert, M., MacDonald, P., dos Remedios, C., Postmus, P., Saripalli, C., Hidalgo, C., Granzier, H., VonkNoordegraaf, A., van der Velden, J., and de Man, F., 2013. "Right Ventricular Diastolic Impairment in Patients With Pulmonary Arterial Hypertension". Circulation, 128(18), pp. 2016-2025.

[35] De Man, F. S., Handoko, M. L., Van Ballegoij, J. J. M., Schalij, I., Bogaards, S. J. P., Postmus, P. E., Van Der Velden, J., Westerhof, N., Paulus, W. J., and VonkNoordegraaf, A., 2012. "Bisoprolol delays progression towards right heart failure in experimental pulmonary hypertension". Circ. Hear. Fail., 5(1), pp. 97-105.

[36] Vonk-Noordegraaf, A., Haddad, F., Chin, K. M., Forfia, P. R., Kawut, S. M., Lumens, J., Naeije, R., Newman, J., Oudiz, R. J., Provencher, S., Torbicki, A., Voelkel, N. F., and Hassoun, P. M., 2013. "Right heart adaptation to pulmonary arterial hypertension: physiology and pathobiology.". J. Am. Coll. Cardiol., 62(25 Suppl), Dec., pp. D22-33.

[37] Hardziyenka, M., Campian, M. E., Verkerk, A. O., Surie, S., Van Ginneken, A. C. G., Hakim, S., Linnenbank, A. C., De Bruin-Bon, H. A. C. M. R., Beekman, L., Van Der Plas, M. N., Remme, C. A., Van Veen, T. A. B., Bresser, P., De Bakker, J. M. T., and Tan, H. L., 2012. "Electrophysiologic remodeling of the left ventricle in pressure overload-induced right ventricular failure". J. Am. Coll. Cardiol., 59(24), pp. 21932202 .

[38] Haddad, F., Hunt, S. A., Rosenthal, D. N., and Murphy, D. J., 2008. Right ventricular function in cardiovascular disease, part I: Anatomy, physiology, aging, and functional assessment of the right ventricle.

[39] Marcus, J. T., Vonk Noordegraaf, a., Roeleveld, R. J., Postmus, P. E., Heethaar, R. M., Van Rossum, a. C., and Boonstra, a., 2001. "Impaired left ventricular filling due to right ventricular pressure overload in primary pulmonary hypertension: noninvasive monitoring using MRI.". Chest, 119(6), pp. 1761-5.

[40] Tanaka, H., Tei, C., Nakao, S., Tahara, M., Sakurai, S., Kashima, T., and Kanehisa, T., 1980. "Diastolic bulging of the interventricular septum toward the left ventricle. An echocardiographic manifestation of negative interventricular pressure gradient between left and right ventricles during diastole.". Circulation, 62(3), pp. 558-563. 
[41] Beyar, R., Dong, S. J., Smith, E. R., Belenkie, I., and Tyberg, J. V., 1993. "Ventricular interaction and septal deformation: a model compared with experimental data.". Am. J. Physiol., 265(6 Pt 2), pp. H2044-H2056.

[42] King, M. E., Braun, H., Goldblatt, a., Liberthson, R., and Weyman, a. E., 1983. "Interventricular septal configuration as a predictor of right ventricular systolic hypertension in children: a cross-sectional echocardiographic study.". Circulation, $\mathbf{6 8}$, pp. 68-75.

[43] Walker, J. C., Ratcliffe, M. B., Zhang, P., Wallace, A. W., Fata, B., Hsu, E. W., Saloner, D., and Guccione, J. M., 2005. "MRI-based finite-element analysis of left ventricular aneurysm.". Am. J. Physiol., 289(2), Aug., pp. H692-700.

[44] Kerckhoffs, R. C. P., 2010. "Patient-specific modeling of the cardiovascular system: Technology-driven personalized medicine". Patient-Specific Model. Cardiovasc. Syst. Technol. Pers. Med., pp. 1-240. 